\title{
Bringing neuroscience to the classroom
}

\section{Is the US National Science Foundation jumping the gun with its plans for education?}

B asic neuroscience and educational theory have, until now, ploughed largely separate academic furrows. But that hasn't stopped overenthusiastic individuals from designing 'brainbased' learning aids - often making healthy profits in the process.

Many of these tools have been built on gross misrepresentations of the science. Take the industry spawned by the idea that there is a 'critical period' for learning in early childhood, when the brain has the highest density of synapses. This ignores evidence that 'pruning' of synaptic connections is a necessary part of brain development.

Now the US National Science Foundation (NSF) is getting serious about the science of learning and its application in the classroom. Cognitive neuroscientists, psychologists, computer scientists and educationalists are being melded into huge collaborative teams (see page 1156).

Hopes are deservedly high. But questions need to be asked about whether the time is ripe for some of the links between basic science and educational practice that are now being proposed.

The computer-based 'cognitive tutors' being developed at Carnegie Mellon University in Pittsburgh are among the most solidly grounded aspects of the NSF initiative. The first tutor, for algebra, has already proved a boon for overstretched teachers. What's more, the Pittsburgh team's strategy for involving teachers in its ongoing research is both innovative and practical.

However, things get a little less convincing when it comes to basic neuroscience. Researchers are planning to use magnetic resonance imaging to 'look under the hood' at the development of skills such as numeracy and reading. It's fascinating stuff, but how the results will inform educational practice remains, for now, largely a matter of speculation. Making meaningful connections between brain activity and behaviour is difficult, even under controlled lab settings.

Brain imaging is seductive, and has an unfortunate tendency to spawn breathless, overreaching media coverage. Care will be needed to ensure that these projects don't encourage ill-informed 'experts' to design yet more pseudoscientific educational tools.

That's not to say that scientific advances can't already help to inform educational policy. For instance, there is now a solid body of evidence that sleep patterns change significantly with age and that, as a result, it makes little sense to wake teenagers up early to go to school, when their attention will be low as a natural
"How 'looking under the hood' at the development of numeracy and reading will inform educational practice remains, for now, largely a matter of speculation." consequence of their daily rhythms. Education authorities and schools are starting to hear this message, and some are adjusting their schedules accordingly.

There's also a strong case for putting the educational tools derived from research in neuroscience to more rigorous empirical tests. For instance, researchers who have evidence that dyslexics have problems with auditory processing have developed a program called Fast ForWord to help them learn to read. But the scientists' company is now marketing the software as a learning aid for children with no specific reading deficits, before they have gathered evidence that it helps anyone other than dyslexics. For now, providing this sort of evidence is where the emphasis should remain.

\section{Crystal clear}

\section{Clarifying the Nature journals' policy on data deposition for chemical structures.}

veryone agrees that data that form the basis of a scientific paper need to be available to readers at the time of publication. But just how raw should such data be? And how much should be released ahead of publication to peer reviewers? These questions can get troublesome when releasing certain data can allow competitors an easy route to results for which the originating researchers have sweated blood. And nowhere is this dilemma more acute than in the data underlying the structures of biological macromolecules and of the complexes that they form.

To labs that solve the structures of proteins and other biological molecules, 'structure factor' files are like the reagents of other fields - material that can be used to enable a variety of experiments. Structure factors are the raw data from which atomic coordinates are derived. As such, they are a key aid for reviewers and readers in verifying a structure.

For many years, Nature and its sibling research journals have required that crystallographers deposit the atomic coordinates of their structures in public data banks at the time of publication. In 2000 the International Union of Crystallography weighed the issues and decided that both coordinates and structure-factor files should be released upon publication, and this is now standard practice.

There is less of a consensus over how to balance the interests of authors and the needs of referees in the peer-review process: should we also require structure-factor files upon submission? After gathering the opinions and insights of a slice of the structure community, Nature and its sibling journals have adopted the following policy.

To help authors maintain control of their data before publication, structure-factor files are "Structure-factor files are not required upon the submission of a manuscript, but editors may request them to aid the review process." not required upon submission of a manuscript. However, editors may request them to aid in the review process. Referees who find that evaluation is dependent on factor files should contact the editor, who will obtain the necessary data set.

We believe that this policy balances appropriate control of data access before publication and the need for rigorous review. 